\title{
Research on Crop Leaf Disease Identification Method Based on LM-BP Neural Network
}

\author{
Fan Tongke* \\ Engineering College of Xi'an International University, Xi'an, China
}

\begin{abstract}
Aiming at the problem of disease diagnosis of large-scale crops, this paper combines machine vision and deep learning technology to propose an algorithm for constructing disease recognition by LM_BP neural network. The images of multiple crop leaves are collected, and the collected pictures are cut by image cutting technology, and the data are obtained by the color distance feature extraction method. The data are input into the disease recognition model, the feature weights are set, and the model is repeatedly trained to obtain accurate results. In this model, the research on corn disease shows that the model is simple and easy to implement, and the data are highly reliable.
\end{abstract}

\section{Introduction}

Machine vision technology is based on image processing technology, using computers to realize human visual functions, involving artificial intelligence, pattern recognition and other disciplines. Machine vision technology is an important research content of agricultural automation and intelligence. In actual production, practical algorithms and techniques are needed to solve the problem. Key technologies for automatic detection and identification of crop diseases include leaf image acquisition techniques, leaf lesion segmentation techniques, preferred techniques for lesion characteristics, and accurate and efficient identification techniques for disease diagnosis. Among them, the collection of leaf images is the basic way to automatically detect and identify crop diseases. In order to alleviate the workload of first-line plant protection personnel, promote the modernization of plant protection in China and the development of agricultural modernization, the research progress and application of machine vision technology in the automatic detection and identification of crop diseases are discussed. On this basis, the problems existing in the current research are analyzed, and the application of machine vision technology in the modern development of plant protection is prospected.

\section{Machine vision based crop disease identification technology}

The crop disease identification process for leaf images mainly includes the following steps:

(1) Selective extraction and real-time extraction from the acquired original blade image to form modeling data and incremental data.

(2) Perform data preprocessing on the two data sets formed in the first step, including image cutting and color distance feature extraction.

(3) using the modeling data of the completed data pre-processing formed by the second step, and classifying the blade images according to experience by an experienced expert, and constructing an expert sampl.

(4) Constructing a classification model using the expert samples of the third step.

(5) Using the fourth step to construct a good classification model for disease classification.

The disease identification flowchart based on leaf image feature extraction is shown in Fig 1.

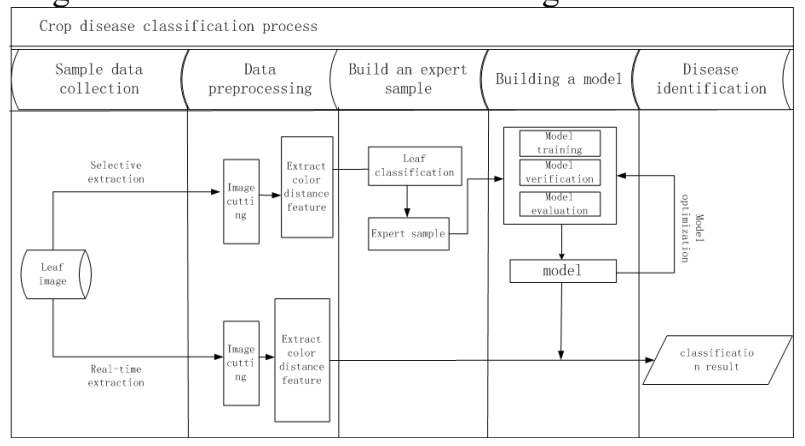

Fig.1 Disease identification flow chart based on leaf image feature extraction

\section{Crop disease image acquisition and pretreatment}

\section{1 leaf image acquisition}

The pictures collected in this paper are all taken in the agricultural research field in Shaanxi Province. The photos are taken by Nikon digital camera, mainly corn

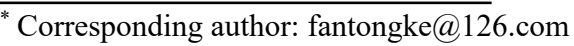


gray spot disease, and the disease period is the early and middle period of crop growth. The captured image is saved in jpg format for convenient calculation. Images are batched into a uniform size via python. In Fig 1 (a) is a disease-free leaf, (b) is a diseased leaf, and (c) is a diseased leaf.

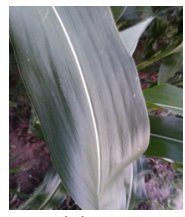

(a)

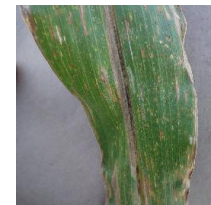

(b)

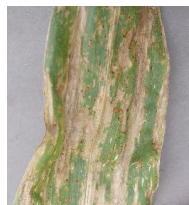

(c)
Fig .2 Image of corn disease leaves

\section{2 image cutting}

After the background color in the image of the collected crop leaves is removed, the difference between the leaf color and the background color is large. At the same time, according to the slender shape of the corn leaf, in order to extract the characteristics of the blade, it is necessary to extract the central part of the blade image to have representative meaning. The image is embodied by extracting an image of $200 * 400$ pixels in the center of the blade image. Let the size of the original image I be $\mathrm{M}^{*} \mathrm{~N}$, then the width of the intercept is from the fix(M/2)-100 pixels to the first fix $(\mathrm{M} / 2)+100$ pixels, and the length is from fix(N/2)- A sub-image of 200 pixels to the first fix $(N / 2)+200$ pixels. Fig 3 (a) is the image before cutting, Fig 3 (b) is the image after cutting. (a)

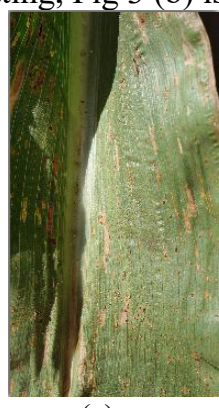

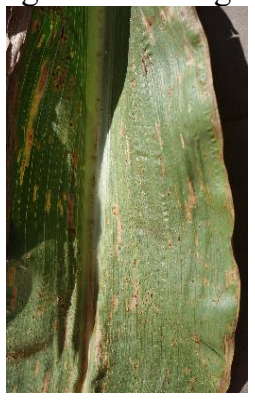

(b)
Fig 3 image cutting

\subsection{Feature Extraction}

Color Matrix (CM) is a simple and effective method for representing image color information. The color distribution information can be described by its moment. The color information is mainly concentrated in the lower moment, so it is generally adopted. The first moment, the second moment, and the third moment of the color represent color features.

The color distance is extracted from the cut image as a color feature of the image.By doing this for all the pictures, you can get the image of the corn disease image and the corresponding category data.

Table 1 Partial data of leaf disease image features and corresponding leaf categories

\begin{tabular}{|c|c|c|c|c|c|c|c|}
\hline number & $\begin{array}{c}\text { First R } \\
\text { channel }\end{array}$ & $\begin{array}{c}\text { First G } \\
\text { channel }\end{array}$ & $\begin{array}{c}\text { First B } \\
\text { channel }\end{array}$ & $\begin{array}{c}\text { Second R } \\
\text { channel }\end{array}$ & $\ldots \ldots$ & $\begin{array}{c}\text { Third B } \\
\text { channel }\end{array}$ & category \\
\hline 1 & 120.35 & 127.01 & 81.25 & 51.81 & $\ldots \ldots$ & 19.70 & 1 \\
\hline 2 & 84.46 & 78.46 & 50.55 & 45.94 & $\ldots \ldots$ & 53.67 & 1 \\
\hline 3 & 111.95 & 120.05 & 85.61 & 61.61 & $\ldots \ldots$ & 49.78 & 1 \\
\hline$\ldots$ & $\ldots \ldots$ & $\ldots \ldots$ & $\ldots \ldots$ & $\ldots \ldots$ & $\ldots \ldots$ & $\ldots \ldots$ & $\ldots$ \\
\hline 90 & 191.75 & 196.91 & 168.94 & 9.00 & $\ldots \ldots$ & -18.03 & 2 \\
\hline 91 & 208.91 & 212.92 & 213.09 & 2.93 & $\ldots \ldots$ & -2.45 & 2 \\
\hline$\ldots$ & $\ldots \ldots$ & $\ldots \ldots$ & $\ldots \ldots$ & $\ldots \ldots$ & $\ldots \ldots$ & $\ldots \ldots$. & $\ldots$ \\
\hline 133 & 154.96 & 171.07 & 108.64 & 13.78 & $\ldots \ldots$ & 23.24 & 3 \\
\hline 134 & 50.31 & 55.25 & 34.81 & 9.88 & $\ldots \ldots$ & 6.82 & 3 \\
\hline
\end{tabular}

\section{Crop disease identification based on LM neural network}

When the number of input nodes of the LM neural network is 9 , the number of output nodes is 5 , the number of hidden layer nodes is 10 , the number of display intervals is 25 , the maximum number of cycles is 1000 , the target error is 0 , the initial error ratio is 0.001 , and the learning growth ratio is 10 , the learning rate reduction ratio is 0.1 , the maximum learning rate is $1 \mathrm{e} 10$, the maximum number of verification failures is 6 , and the minimum error gradient is 1e-7. The confusion matrix of the training sample modeling is shown in Fig.4. The confusion matrix of the output results is shown in
Fig. 5. The classification accuracy rate is $88 \%$, indicating that the classification model has better effect on the classification of new pest and disease images. 


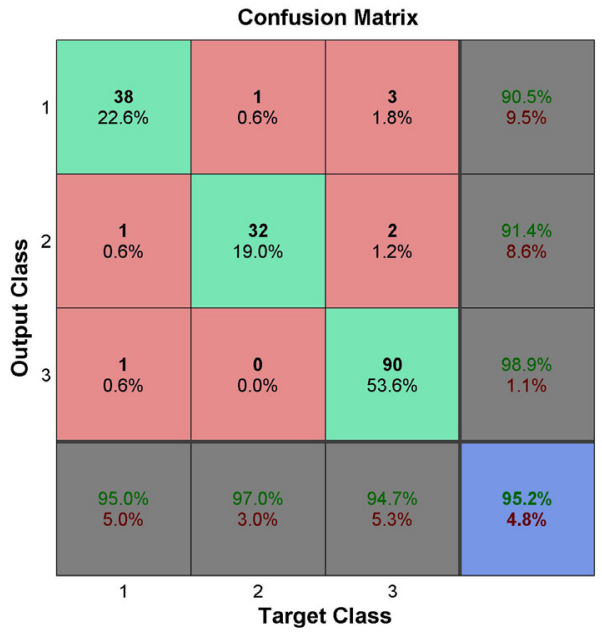

Fig 4 Modeling confusion matrix

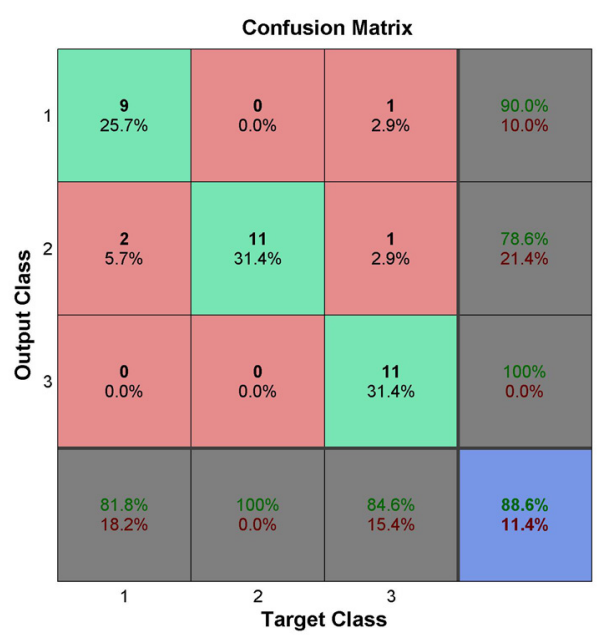

Fig 5 Confusion matrix of prediction results

\section{Conclusion}

In this paper, the research on disease identification of corn leaf images focuses on the application of image cutting technology and the application of Levenbery Marquardt optimization algorithm in BP neural network. The image is cut by image cutting technology, and the color distance feature extraction method is used to obtain the data. The neural network in the data mining algorithm is used to establish the corn leaf image for disease identification model, and the data is input into the model, and the model is repeated. Training to get accurate results later. The research of this technology can bring a lot of convenience to the planting industry and is more conducive to the better development of the planting industry. Through this technology, subjective observation errors due to eye observation can be avoided, and a large amount of manpower and material resources are saved, which is simple and practical and convenient for popularization and application.

\section{Acknowledgement}

This research is supported by the Key Research and
Development Program of Shaanxi Province in 2019 under grant number 2019NY-055. The authors wish to thank the anonymous reviewers for their valuable comments.

\section{References}

1. Zhang Liangjun. MATLAB data analysis and mining practice $[\mathrm{M}]$. Beijing: Mechanical Industry Press, 2015, 256 - 278.

2. Xu Qiao. Research and development of personalized information filtering system based on vector space model. Computer and Digital Engineering, 2014. 10(2): 1940-1944.

3. Lai Junchen, Li Shaokun, Ming Bo, et al. Progress in Machine Vision Diagnosis of Crop Diseases[J]. Scientia Agricultura Sinica, 2009, 42(4): 1215-1221.

4. Wang Xianfeng, Zhang Shanwen, Wang Zhen, et al. Identification method of cucumber disease based on leaf image and environmental information[J]. Transactions of the Chinese Society of Agricultural Engineering, 2014, 30(14):148-153.

5. Li Zongru. Research on Apple Disease Identification Technology Based on Image Analysis [D]. Northwest A\&F University, 2010.

6. Tian Jie, Han Dong, Hu Qiuxia, et al. Color image segmentation of wheat diseases based on PCA and Gaussian mixture model[J]. Transactions of the Chinese Society of Agricultural Machinery, 2014, 45(7):267-271.

7. Su Hengqiang. Design and implementation of image recognition system for corn disease [D]. Jilin University, 2010.

8. $\mathrm{Xu}$ Liangfeng, $\mathrm{Xu}$ Xiaobing, $\mathrm{Hu} \mathrm{Min}$, et al. Identification of maize leaf diseases based on multi-classifier fusion[J]. Transactions of the Chinese Society of Agricultural Engineering, 2015, 31(14): 194-201.

9. Amlekar M, Manza R R, Yannawar P, et al. Leaf Features Based Plant Classification Using Artificial Neural Network [J]. IBMRD's Journal of Management \& Research, 2014, 3(1): 224-232.

10. Tao Huawei, Zhao Li, Yan Ji, et al. Identification methods of fruit and vegetable based on color and texture characteristics[J]. Transactions of the Chinese Society of Agricultural Engineering, 2014(16):305-311.

11. Lu Guanming, He Jiali, Yan Jingjie, et al. A Convolutional Neural Network for Facial Expression Recognition[J]. Journal of Nanjing University of Posts and Telecommunications(Natural Science Edition), 2016, 36(1): 16-22.

12. Goodfellow I J, Wardefarley D, Mirza M, et al. Maxout Networks [J]. Computer Science, 2013: 1319-1327. 